\title{
Comparison of the effect of individual dietary counselling and of standard nutritional care on weight loss in patients with head and neck cancer undergoing radiotherapy
}

\author{
Manon G. A. van den Berg ${ }^{1}$, Ellen L. Rasmussen-Conrad ${ }^{1}$, Koko H. Wei ${ }^{1,2}$, Heleen Lintz-Luidens ${ }^{3}$, \\ Johannes H. A. M. Kaanders ${ }^{3}$ and Matthias A. W. Merkx ${ }^{4}$ \\ ${ }^{1}$ Department of Gastroenterology and Hepatology - Dietetics (367), Radboud University Nijmegen Medical Centre, PO Box 9101 , \\ $6500 \mathrm{HB}$ Nijmegen, The Netherlands \\ ${ }^{2}$ Department of Human Nutrition, Wageningen University, Wageningen, The Netherlands \\ ${ }^{3}$ Department of Radiation Oncology, Radboud University Nijmegen Medical Centre, Nijmegen, The Netherlands \\ ${ }^{4}$ Department of Oral and Maxillofacial Surgery, Radboud University Nijmegen Medical Centre, Nijmegen, The Netherlands
}

(Received 11 August 2009 - Revised 15 March 2010 - Accepted 16 March 2010 - First published online 5 May 2010)

\begin{abstract}
Clinical research shows that nutritional intervention is necessary to prevent malnutrition in head and neck cancer patients undergoing radiotherapy. The objective of the present study was to assess the value of individually adjusted counselling by a dietitian compared to standard nutritional care (SC). A prospective study, conducted between 2005 and 2007, compared individual dietary counselling (IDC, optimal energy and protein requirement) to SC by an oncology nurse (standard nutritional counselling). Endpoints were weight loss, BMI and malnutrition (5\% weight loss/month) before, during and after the treatment. Thirty-eight patients were included evenly distributed over two groups. A significant decrease in weight loss was found 2 months after the treatment $(P=0.03)$ for IDC compared with SC. Malnutrition in patients with IDC decreased over time, while malnutrition increased in patients with SC $(P=0.02)$. Therefore, early and intensive individualised dietary counselling by a dietitian produces clinically relevant effects in terms of decreasing weight loss and malnutrition compared with SC in patients with head and neck cancer undergoing radiotherapy.
\end{abstract}

Dietary counselling: Malnutrition: Head and neck cancer: Radiotherapy

Malnutrition is a substantial problem in Dutch health care. This applies for more than one in five patients ${ }^{(1)}$. In head and neck cancer patients, malnutrition, specified as unintended weight loss $\geq 5 \%$ in 1 month and/or $\geq 10 \%$ in 6 months, has been reported in 30-50\%, particularly in those with squamous cell carcinomas in the oropharyngeal and hypopharyngeal areas $^{(2,3)}$. The most common treatments for these patients include surgery, radiotherapy, chemotherapy or a combination of these ${ }^{(4,5)}$. The risk of nutritional deterioration is increased during the actual treatment. Radiotherapy and/or chemoradiation induce morbidity; symptoms such as mucositis, impaired swallowing function, declined eating ability, xerostomia, dysgeusia, nausea and vomiting may limit oral intake, and inevitably result in unintended weight loss during radiotherapy and for a prolonged period after the treatment ${ }^{(2,6-9)}$.

Nutritional depletion in these patients reduces their tolerance to treatment. Malnutrition in head and neck cancer patients was significantly correlated with an increased risk of infections in patients undergoing surgery and the occurrence of major postoperative complications ${ }^{(3,10)}$. In addition, higher mortality and morbidity rates, shorter failure-free survival $^{(11-14)}$ and poorer quality of life in all therapies including radiotherapy have been reported ${ }^{(15-17)}$. More specifically, unintended weight loss was found to be associated with a higher rate of recurrence and second primary tumours of the oral cavity and oropharynx after radiotherapy ${ }^{(13)}$. Weigh loss was found to be associated with reduced kidney function during cisplatin-containing chemoradiotherapy ${ }^{(14)}$. Even though no cause-effect relationships were established in the study of Lin et al., the findings emphasise the importance of intensive supportive measures beyond standard nutrition and hydration intervention.

It is therefore important to maintain an optimal nutritional status for patients through nutritional intervention during oncological treatment.

Several studies suggested that early and intensive nutritional intervention during radiotherapy may be beneficial in terms of decreasing the impact of side effects, decreasing unintended weight loss, and improving dietary intake, quality of life and treatment tolerance ${ }^{(6,18-21)}$. According to published nutritional management guidelines for head and neck cancer patients, nutritional interventions must be initiated before

Abbreviations: IDC, individual dietary counselling; SC, standard nutritional care.

* Corresponding author: Manon G. A. van den Berg, fax +31 24 3618889, email m.vandenberg@ dieet.umcn.nl 


\begin{tabular}{|c|c|c|c|}
\hline Diagnosis & Treatment & \begin{tabular}{|c|}
$\begin{array}{c}\text { Early } \\
\text { rehabilita- } \\
\text { tion }\end{array}$ \\
\end{tabular} & Rehabilitation \\
\hline 1(Baseline) & & 3 & \\
\hline 4 weeks & 6 weeks & 2 weeks & 8 weeks \\
\hline
\end{tabular}

Fig. 1. Data collection: weight in $\mathrm{kg}(1-5)$ measured during diagnosis, treatment, early rehabilitation and rehabilitation.

and continued during and after cancer treatment, and the implementation may be more successful if a dietitian is involved $^{(22-24)}$.

Although many studies demonstrate the benefits of full nutritional intervention programme, including dietary counselling, there is little evidence for the potential added value of a professional dietitian's support. In the current health care system, there is a need to justify resources and to demonstrate the effects of individual dietary counselling.

The present study was designed to evaluate the effect of individual dietary counselling (IDC) by a dietitian for patients with oral cavity, oropharyngeal or hypopharyngeal cancer undergoing radiotherapy before, during and after the treatment. The objective was to investigate whether individualised dietary counselling by a dietitian would better maintain a patient's body weight, and thus prevent malnutrition compared to standard nutritional care (SC).

\section{Experimental methods}

\section{Study design}

A prospective clinical study on the impact of dietary counselling on unintended weight loss, BMI $\left(\mathrm{kg} / \mathrm{m}^{2}\right)$ and malnutrition in patients with head and neck carcinomas was carried out between January 2005 and February 2007 at the Radboud University Nijmegen Medical Centre. Patients with primary squamous cell carcinoma in the oral cavity, oropharynx or hypopharynx, age $\geq 18$ years, stages II-IV (International Union Against Cancer TNM classification of malignant tumours) were included ${ }^{(25)}$. Patients were treated depending on stage, location of the tumour and general health conditions by radiotherapy, combined surgery, and radiotherapy or concomitant radio-chemotherapy in accordance with the guidelines of the Dutch Cooperative Head and Neck Oncology Group ${ }^{(4,5)}$.

From 2005 till 2007, dietary counselling was given to all patients by a dietitian before the start of radiotherapy.

Next, patients were assigned to one of the intervention groups based on their postal code. One group continued to receive IDC by a dietitian throughout the entire treatment and rehabilitation period (IDC). The other group was passed on to trained nurses for SC during radiotherapy and thereafter.

At baseline (the first visit to the outpatient clinic at the departments of Otorhinolaryngology or Oral and Maxillofacial Surgery), patients' characteristics including age, sex, actual body weight $(\mathrm{kg})$ and height $(\mathrm{m})$ were registered. Parameters studied from baseline through to rehabilitation included body weight $(\mathrm{kg})$, BMI $\left(\mathrm{kg} / \mathrm{m}^{2} ;<18.5=\right.$ underweight; $18 \cdot 5-25=$ normal range; $\geq 25=$ overweight; $\geq 30=$ obese) ${ }^{(26)}$ and malnutrition (unintended weight loss $\geq 5 \%$ in 1 month and/or $\geq 10 \%$ in 6 months $)^{(3,27)}$. Body weight was measured on a calibrated one decimal Seca scale (delta $\mathrm{nr}$ 707; Seca, Hamburg, Germany) wearing lightweight clothing under the same conditions.

Measurements were taken at five pre-determined time points for each treatment modality (Fig. 1).

\section{Nutrition policy}

Individual dietary counselling. IDC in the present study is defined as individualised and intensive dietary counselling by dietitians focused on maintaining and/or improving a patient's energy and protein intake. Counselling is based on four nutritional guidelines ${ }^{(4,5,28,29)}$.

These guidelines describe treatment-related symptoms that influence dietary intake, and offer advice on how to deal with these symptoms, including dietary counselling strategies, when dietary counselling is necessary and when to start tube feeding (nasogastric/gastrostomy). To meet the specific nutritional goals for each patient, individual directions for dietary counselling were given. The patient's energy requirement was estimated at $>125 \mathrm{~kJ}$ to a maximum of $167 \mathrm{~kJ}$ $(>30-\leq 40 \mathrm{kcal})$ per $\mathrm{kg}$ of actual body weight depending on activities, metabolic stress, abnormal losses and treatment. The protein requirement was normally estimated at $>1.0$ to a maximum of $1.5 \mathrm{~g}$ of protein per $\mathrm{kg}$ ideal body weight depending on bedsores or other inflammatory disorders which require extra protein intake. Ideal body weight was estimated as follows: (1) if a patient was underweight (BMI $\left.<20 \mathrm{~kg} / \mathrm{m}^{2}\right)$ : ideal weight $(\mathrm{kg})=20 \mathrm{~kg} / \mathrm{m}^{2} \times$ height $\left(\mathrm{m}^{2}\right)$; (2) if a patient was overweight $\left(\right.$ BMI $\left.>25 \mathrm{~kg} / \mathrm{m}^{2}\right)$ : ideal weight $(\mathrm{kg})=25$ $\mathrm{kg} / \mathrm{m}^{2} \times$ height $\left(\mathrm{m}^{2}\right)$; a patient having a BMI of $20-25 \mathrm{~kg} / \mathrm{m}^{2}$ was considered to be of ideal weight. IDC involved the prescription of a therapeutic diet with regular food which was adjusted to the individual's usual diet, thereby recognising personal eating patterns, feasible consistency and preferences. The dietitian would also take other relevant factors into consideration, namely the need for alleviation or arrest of local symptoms, as well as psychological factors and digestive and absorptive capacity. The prescription included the type, amount and frequency of feeding, and specified the energetic/ protein level that had to be attained. If a patient's oral intake continued to diminish, energy- and protein-rich oral nutrition supplements were supplied separately or together with normal meals. Supplements (high energetic drinks) were offered to patients in their preferred flavours, and they were instructed to use them as drinks to be consumed between meals or in combination with snacks between meals. When all the abovementioned methods were insufficient to maintain the required energy and protein intake for patients, nutritional intake was achieved through tube feeding. Gastrostomy tubes were used in cases where tube feeding was expected to be necessary for a 
period longer than 6 weeks. From the start of the radiotherapy until at least 2 months after the treatment, patients were checked twice before and at least once a week during and after the radiotherapy. The dietetic consultation consisted of twelve to fifteen visits, but it could be arranged more frequently when requested. The dietitian was available to answer any queries or provide more information. When necessary, dietetic domiciliary care was arranged for the subsequent period.

Standard nutritional care by a nurse. Patients were seen twice by a dietitian before radiotherapy (IDC policy) and when radiotherapy started they were passed on to SC by a nurse. This is as follows: body weight of each patient was measured at least once a week on a calibrated scale wearing lightweight clothing.

Nutritional advice was based on a guideline specifically focused on treatment symptoms such as pain, nausea, xerostomia, mucositis and dysphagia, and how to deal with nutrition ${ }^{(29)}$.

Interventions were mostly focused on evaluating pain due to radiation mucositis and adjusting pain medication. When nutritional intake seemed insufficient, advice on high energetic liquid nutrition and/or high kiloenergetic/protein supplements was given. If the above-mentioned methods failed to work, a nasogastric tube was used for patients who had lost more than $10 \%$ of their body weight. Gastrostomy tubes were used in cases where tube feeding was expected to be necessary for a period exceeding 6 weeks.

The frequency of nutritional advice was at least once a week, but this increased when more problems occurred (daily, if necessary, depending on the treatment phase and symptoms). Patients were seen at the outpatient clinic or contacted by phone up to a minimum of 3-4 months after the treatment. Trained nurses offered patients support during and after the treatment in all areas concerning illness and side effects such as nutritional, psychological and skin problems, pain and constipation. This support was supervised by a radiation oncologist.

\section{Statistical analysis}

Data processing and statistical analysis were performed using Statistical Package for Social Sciences version 16.0 (SPSS, Inc., Chicago, IL, USA).

The percentage of unintended weight loss was calculated using baseline weight as a reference point.

A descriptive statistical analysis was performed for the two groups of patients. Differences in the distribution of patients' characteristics were evaluated by $\chi^{2}$ test. Differences in the mean change in outcome between and within groups were tested by independent and dependent Student's $t$ tests, respectively. Fisher's exact probability test was performed to test the difference in malnutrition between the treatment groups. The level of statistical significance was set at $P<0.05$, two-sided.

The present study was conducted according to the guidelines laid down in the Declaration of Helsinki, and all procedures involving human subjects/patients were approved by the Committee on Research Involving Human Subjects of the Radboud University Nijmegen Medical Centre. Written informed consent was obtained from all subjects/patients.
Table 1. Baseline characteristics of the patients (Mean values with their standard errors)

\begin{tabular}{|c|c|c|c|c|}
\hline \multirow[b]{2}{*}{ Variable } & \multicolumn{2}{|c|}{ IDC ( $n$ 20) } & \multicolumn{2}{|c|}{$\mathrm{SC}(n 18)$} \\
\hline & Mean & SE & Mean & SE \\
\hline Age (years) & $63 \cdot 8$ & 1.9 & $61 \cdot 6$ & $2 \cdot 1$ \\
\hline Height $(m)$ & 1.70 & 0.00 & 1.71 & 0.02 \\
\hline Sex (male) & $14 \cdot 0$ & & 8.0 & \\
\hline Weight (kg) & $69 \cdot 3$ & 3.4 & 71.0 & 3.8 \\
\hline BMI $\left(\mathrm{kg} / \mathrm{m}^{2}\right)^{*}$ & 23.9 & $1 \cdot 1$ & $24 \cdot 0$ & 0.9 \\
\hline$<18.5$ & 3.0 & & 1.0 & \\
\hline $18 \cdot 5-25 \cdot 0$ & $10 \cdot 0$ & & $12 \cdot 0$ & \\
\hline$>25.0$ & $7 \cdot 0$ & & $5 \cdot 0$ & \\
\hline \multicolumn{5}{|l|}{ T stage } \\
\hline T2 & $6 \cdot 0$ & & $11 \cdot 0$ & \\
\hline T3 & $7 \cdot 0$ & & 4.0 & \\
\hline T4 & $7 \cdot 0$ & & $3 \cdot 0$ & \\
\hline \multicolumn{5}{|l|}{$\mathrm{N}$ stage } \\
\hline No & $9 \cdot 0$ & & 5.0 & \\
\hline$N \geq 1$ & $11 \cdot 0$ & & $13 \cdot 0$ & \\
\hline Tumour site & $10 \cdot 0$ & & 3.0 & \\
\hline \multicolumn{5}{|l|}{ Oral cavity } \\
\hline Oropharynx & $7 \cdot 0$ & & $12 \cdot 0$ & \\
\hline Hypopharynx & 3.0 & & $3 \cdot 0$ & \\
\hline \multicolumn{5}{|l|}{ Treatment } \\
\hline Radiotherapy & $6 \cdot 0$ & & $7 \cdot 0$ & \\
\hline Chemo/radiotherapy & $6 \cdot 0$ & & 5.0 & \\
\hline Surgery/radiotherapy & 8.0 & & $6 \cdot 0$ & \\
\hline
\end{tabular}

IDC, individual dietary counselling; SC, standard nutritional care by a nurse.

${ }^{\star} \mathrm{BMI}\left(\mathrm{kg} / \mathrm{m}^{2}\right)$ : $<18.5$, underweight; 18.5-25.0, healthy weight; $>25 \cdot 0$, overweight $(\mathrm{WHO})^{(26)}$.

\section{Results}

A total of thirty-eight patients met the inclusion criteria. The IDC group included twenty patients, and the SC group consisted of eighteen patients. Table 1 summarises baseline characteristics of the two groups. There were no significant differences between age, height, sex, weight, BMI, T stage, $\mathrm{N}$ stage, tumour site and treatment.

\section{Percentage of unintended weight loss}

The percentage weight changes for the two groups are shown in Fig. 2.

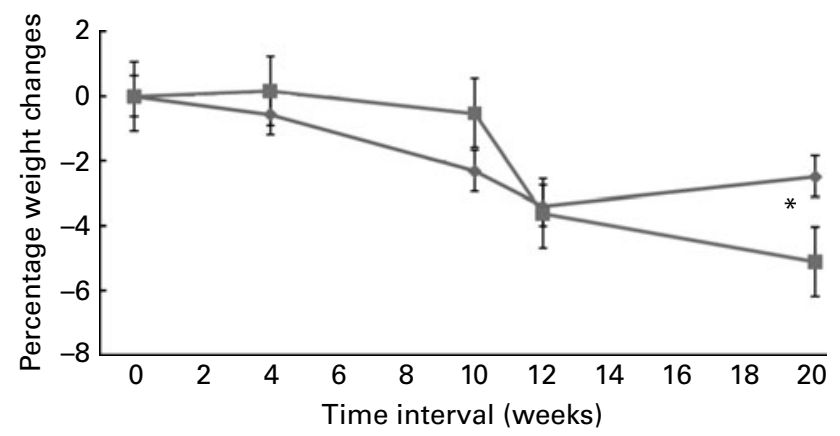

Fig. 2. Percentage of unintended weight loss as a function of time (means with their standard errors), with baseline as reference. IDC, individual dietary counselling $(\neg-, n 20)$; SC, standard nutritional care by a nurse $(-, n$ 18). * Mean values were significantly different between SC and IDC groups $(P=0.03)$. 
The IDC group had a maximum of $3 \%$ unintended weight loss 2 weeks after the treatment. This was approximately the same for the SC group. Two months after the treatment, the IDC group started to gain weight (1\% weight gain), while the SC group continued to lose $1.5 \%$ weight. Two months after the treatment, weight loss was significantly different between SC and IDC groups $(P=0 \cdot 03)$.

\section{$B M I$}

Most of the patients in IDC and SC groups had a normal BMI $\left(18 \cdot 5 \mathrm{~kg} / \mathrm{m}^{2} \leq \mathrm{BMI}<25 \mathrm{~kg} / \mathrm{m}^{2}\right)$ throughout the study (Fig. 3). No patients were underweight $\left(\mathrm{BMI}<18.5 \mathrm{~kg} / \mathrm{m}^{2}\right)$. BMI for the IDC and SC groups did not differ significantly at any of the time points.

\section{Malnutrition}

Table 2 displays the prevalence of malnutrition from diagnosis until 2 months after the treatment. Before treatment, four of twenty patients in the IDC group and three of eighteen patients in the SC group were malnourished. During the treatment period, the IDC and SC groups remained stable. Two weeks after the treatment, there was a significant difference in malnutrition between the IDC (0/20) and SC $(5 / 18)$ groups $(P=0 \cdot 02)$.

Two months after the treatment, the highest prevalence of malnutrition was seen for the SC group (3/18).

The prevalence of malnutrition by $\mathrm{T}$ stages is shown in Table 3. No significant differences were found between the $\mathrm{T}$ stages.

\section{Discussion}

Malnutrition, defined as $\geq 5 \%$ of unintended weight loss/ month or $\geq 10 \%$ of unintended weight loss $/ 6$ months, is the most commonly used parameter in clinical practice for head and neck cancer ${ }^{(3,27)}$. In the literature, the prevalence of malnutrition is associated with higher mortality and morbidity rates, shorter failure-free survival and poorer quality of life among radiotherapy patients ${ }^{(11-17)}$. It is therefore essential to control unintended weight loss/malnutrition and maintain an efficient nutritional status for patients. The present study demonstrates the beneficial effect of IDC on weight change, BMI and malnutrition for patients with oral cavity,

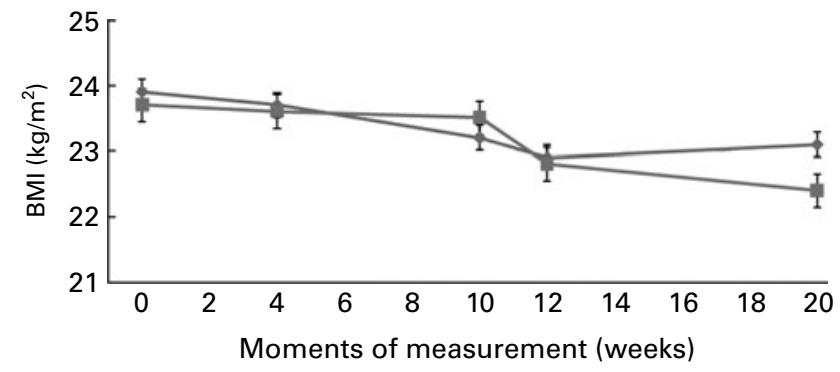

Fig. 3. BMI as a function of time (means with their standard errors), with baseline as reference. $\mathrm{BMI}$ : $<18.5=$ underweight; $18.5-25=$ healthy weight; $>25=$ overweight $(\mathrm{WHO})^{(26)}$. IDC, individual dietary counselling $(\neg, n 20)$; SC, standard nutritional care by a nurse $(-, n 18)$.
Table 2. Prevalence of malnutrition* in individual dietary counselling (IDC) and standard nutritional care by a nurse (SC) groups from diagnosis until rehabilitation

\begin{tabular}{lccc}
\hline & \multicolumn{2}{c}{$\begin{array}{c}\text { Number of patients } \\
\text { per nutrition intervention }\end{array}$} & \\
\cline { 2 - 3 } Interval & ICD $(n 20)$ & SC $(n$ 18) & Total $(n$ 38) \\
\hline Diagnosis & 4.0 & 3.0 & 7.0 \\
Treatment & 3.0 & 4.0 & 7.0 \\
Early rehabilitation & $0.0 \dagger$ & $5.0 \dagger$ & 5.0 \\
Rehabilitation & 1.0 & 3.0 & 4.0 \\
\hline
\end{tabular}

*Malnutrition was defined as 'unintended weight loss $\geq 5 \%$ within 1 month'.

†Prevalence of malnutrition in IDC and SC groups was significantly different $(P<0.05)$.

oropharyngeal and hypopharyngeal cancers treated with radiotherapy compared to SC.

Two different groups (IDC and SC) were compared in the present study. Patients' characteristics were evenly distributed. Treatment-induced side effects of patients receiving radiotherapy to the head and neck area peaked at the end of the treatment course and continued for 2 or more weeks after the treatment ${ }^{(20)}$. This is reflected by a sharp weight reduction during this period, which continued until 2 months after the treatment, specifically in the SC group, while the IDC group started gaining weight 2 weeks after the radiotherapy. There was a significant difference in unintended weight loss 2 months after the treatment for the SC group compared with the IDC group.

The IDC group started with the same proportion of patients with malnutrition (4/20) v. SC (3/18) at diagnosis where malnutrition in the SC group increased over time. A significant difference in the prevalence of malnutrition for the early rehabilitation period was seen between the groups $(P=0.02)$, which affirms that dietary counselling recovers malnutrition where SC increases malnutrition.

While looking at the overall results of the present study, the most striking differences are observed in the (early) rehabilitation period. Two months after the treatment, weight and BMI in the SC group were still declining. In future research, a longer follow-up is therefore required to determine the nadir of weight loss and the duration of full recovery. Also, more data about nutritional status should be included such as a comprehensive nutritional assessment tool (e.g. subjective global assessment or patient-generated subjective global assessment), anthropometric measurements (e.g. bioimpedance and/or handgrip) and food intake (e.g. dietary history or FFQ). Besides, it should be useful to include other endpoints such

Table 3. Prevalence of malnutrition* per $\mathrm{T}$ stage from diagnosis until rehabilitation

\begin{tabular}{|c|c|c|c|c|}
\hline \multirow[b]{2}{*}{ Interval } & \multicolumn{3}{|c|}{ Number of patients per $\mathrm{T}$ stage } & \multirow[b]{2}{*}{ Total $(n 38)$} \\
\hline & T2 $(n 17)$ & T3 $(n 11)$ & $\mathrm{T} 4(n$ 10) & \\
\hline Diagnosis & $2 \cdot 0$ & $3 \cdot 0$ & $2 \cdot 0$ & $7 \cdot 0$ \\
\hline Treatment & $3 \cdot 0$ & $3 \cdot 0$ & $1 \cdot 0$ & $7 \cdot 0$ \\
\hline Early rehabilitation & $5 \cdot 0$ & $0 \cdot 0$ & $0 \cdot 0$ & $5 \cdot 0$ \\
\hline Rehabilitation & $3 \cdot 0$ & $1 \cdot 0$ & $0 \cdot 0$ & $4 \cdot 0$ \\
\hline
\end{tabular}

* Malnutrition was defined as 'unintended weight loss $\geq 5 \%$ within 1 month'. 
as quality of life, mortality, response to treatment and length of hospital stay.

These results indicate that IDC significantly contributes to maintaining and improving weight loss and malnutrition of head and neck cancer patients undergoing radiotherapy. This is an important clinically relevant finding which confirms the research objective of the present study.

A randomised study performed by Ravasco et $a l^{(6)}$ produced similar results. The study demonstrated that individualised dietary counselling (based on regular foods) for head and neck cancer patients undergoing radiotherapy is the most effective way of improving patients' nutritional intake, nutritional status and quality of life by the end of the treatment until 3 months after the treatment.

However, Ravasco used Ottery's Subjective Global Assessment (unscored patient-generated subjective global assessment) to determine malnutrition. In this system, unintended weight loss is one of the deciding factors besides symptoms, metabolic stress and subcutaneous fat.

A randomised controlled trial by Isenring et al. ${ }^{(20,21)}$ showed that dietary counselling using the American Dietetic Association - Medical Nutrition Therapy radiation oncology protocol resulted in a significant reduction in unintended weight loss from the start of radiotherapy until 3 months after the treatment compared with their standard practice (which consisted of general nutritional advice by a nurse and a booklet). Furthermore, the present study revealed an improvement in nutritional status (patient-generated subjective global assessment), dietary intake and quality of life in the dietary counselling group when compared with the standard practice. This is the first prospective randomised controlled trial. A limitation of the present study is that two different tumour locations were involved (gastrointestinal and head and neck cancers).

Dawson et al. ${ }^{(30)}$ reported a significant reduction in unintended weight loss in oral cancer patients undergoing combined modality treatment (surgery + radiotherapy) with increased dietary supervision compared with standard dietary counselling after revising their dietary protocol. However, this was not compared to nutritional standard care without dietary counselling. It is interesting to note that while there have been only a handful of studies in this area by different research teams, they all have demonstrated benefits of dietary counselling like the present study.

To summarise, early and intensive IDC produces clinically relevant effects in terms of decreasing unintended weight loss and malnutrition compared with SC in patients with head and neck cancer undergoing radiotherapy. This is especially the case early after the treatment and during the rehabilitation period.

The guideline used by the Radboud University Nijmegen Medical Centre Department of Dietetics for head and neck cancer patients is a useful guide in supplying the level of dietary support required ${ }^{(4,5,28,29)}$. In clinical practice, patients should receive regular and individualised dietary counselling from diagnosis until at least 2 months after radiotherapy and probably longer.

\section{Acknowledgements}

The present research received no specific grant from any funding agency in the public, commercial or non-profit sector. All authors hereby declare that no financial or personal interest is involved which may bias or influence the present study. All authors contributed to the concept and design of the present study, acquisition, analysis and interpretation of data. All authors approved the final version of the present manuscript. We are grateful to Jeanne de Vries (Department of Human Nutrition, Wageningen University, The Netherlands) for the useful discussions.

\section{References}

1. Meijers JMM, Schols JMGA, van Bokhorst-de van der Schueren MA, et al. (2009) Malnutrition prevalence in The Netherlands: results of the annual Dutch national prevalence measurement of care problems. Br J Nutr 101, 417-423.

2. van den Berg MG, Rasmussen-Conrad EL, Gwasara GM, et al. (2006) A prospective study on weight loss and energy intake in patients with head and neck cancer, during diagnosis, treatment and revalidation. Clin Nutr 25, 765-772.

3. van Bokhorst-de van der Schueren MA, van Leeuwen PA, Sauerwein HP, et al. (1997) Assessment of malnutrition parameters in head and neck cancer and their relation to postoperative complications. Head Neck 19, 419-425.

4. CBO (2004) Richtlijn Mondholte-en Oropharynxcarcinoom (Directive Oral Cavity and Oropharynxcarcinoma), 1st ed. Alphen aan den Rijn: Van Zuiden Communications B.V.

5. Oncoline (2007) Richtlijn Hypopharynx carcinoom (Hypopharyngeal cancer guidelines). http://www.oncoline.nl/index. php?language $=$ en

6. Ravasco P, Monteiro-Grillo I, Marques VP, et al. (2005) Impact of nutrition on outcome: a prospective randomized controlled trial in patients with head and neck cancer undergoing radiotherapy. Head Neck 27, 659-668.

7. Newman LA, Vieira F, Schwiezer V, et al. (1998) Eating and weight changes following chemoradiation therapy for advanced head and neck cancer. Arch Otolaryngol Head Neck Surg 124, 589-592.

8. Munshi A, Pandey MB, Durga T, et al. (2003) Weight loss during radiotherapy for head and neck malignancies: what factors impact it? Nutr Cancer 47, 136-140.

9. Lees J (1999) Incidence of weight loss in head and neck cancer patients on commencing radiotherapy treatment at a regional oncology centre. Eur J Cancer Care (Engl) 8, 133-136.

10. Van Bokhorst-De Van der Schuer MA, von Blomberg-van der Flier BM, Riezebos RK, et al. (1998) Differences in immune status between well-nourished and malnourished head and neck cancer patients. Clin Nutr 17, 107-111.

11. van Bokhorst-de van der Schuer, van Leeuwen PA, Kuik DJ, et al. (1999) The impact of nutritional status on the prognoses of patients with advanced head and neck cancer. Cancer 86, 519-527.

12. Liu SA, Tsai WC, Wong YK, et al. (2006) Nutritional factors and survival of patients with oral cancer. Head Neck 28, 998-1007.

13. Nguyen TV \& Yueh B (2002) Weight loss predicts mortality after recurrent oral cavity and oropharyngeal carcinomas. Cancer 95, 553-562.

14. Lin A, Jabbari S, Worden FP, et al. (2005) Metabolic abnormalities associated with weight loss during chemoirradiation of head-and-neck cancer. Int J Radiat Oncol Biol Phys 63, 1413-1418.

15. Petruson KM, Silander EM \& Hammerlid EB (2005) Quality of life as predictor of weight loss in patients with head and neck cancer. Head Neck 27, 302-310.

16. Campbell BH, Spinelli K, Marbella AM, et al. (2004) Aspiration, weight loss, and quality of life in head and neck cancer survivors. Arch Otolaryngol Head Neck Surg 130, 1100-1103. 
17. van den Berg MG, Rasmussen-Conrad EL, van Nispen L, et al. (2008) A prospective study on malnutrition and quality of life in patients with head and neck cancer. Oral Oncol 44, $830-837$.

18. Rabinovitch R, Grant B, Berkey BA, et al. (2006) Impact of nutrition support on treatment outcome in patients with locally advanced head and neck squamous cell cancer treated with definitive radiotherapy: a secondary analysis of RTOG trial 90-03. Head Neck 28, 287-296.

19. Gonçalves Dias MC, de Fátima Nunes Marucci M, Nadalin W, et al. (2005) Nutritional intervention improves the caloric and proteic ingestion of head and neck cancer patients under radiotherapy. Nutr Hosp 20, 320-325.

20. Isenring EA, Capra S \& Bauer JD (2004) Nutrition intervention is beneficial in oncology outpatients receiving radiotherapy to the gastrointestinal or head and neck area. Br J Cancer 91, $447-452$.

21. Isenring EA, Bauer JD \& Capra S (2007) Nutrition support using the American Dietetic Association medical nutrition therapy protocol for radiation oncology patients improves dietary intake compared with standard practice. J Am Diet Assoc 107, 404-412.

22. Wood K (2005) Audit of nutritional guidelines for head and neck cancer patients undergoing radiotherapy. J Hum Nutr Diet 18, 343-351.
23. Meuric J, Garabige V, Blanc-Vincent MP, et al. (1999) Good clinical practice in nutritional management of head and neck cancer patients. Bull Cancer 86, 843-854.

24. CBO (2004) Voeding en dieettherapie. Richtlijn Mondholte-en Oropharynxcarcinoom (Nutrition and Diet Therapy. Directive Oral Cavity and Oropharynxcarcinoma), 1st ed., pp. 187-190. Alphen aan den Rijn: Van Zuiden Communications B.V.

25. Mc Kee PH (2002) Wolf Comprehensive Tumour Terminology Handbook, 6th ed., pp. 384. New York: John Wiley \& Sons.

26. WHO (2004) BMI. http://www.who.int/bmi/index.jsp?introPage =intro_3.html

27. Ravasco P, Monteiro-Grillo I, Vidal PM, et al. (2003) Nutritional deterioration in cancer: the role of disease and diet. Clin Oncol (R Coll Radiol) 15, 443-450.

28. Vogel-Boezeman J (editor) (2003) Elsevier gezondheidszorg, Dieet behandelingsrichtlijnen. Kanker (Elsevier Health, Diet Treatment Guidelines. Cancer), 1st ed., pp. 1-36. Maarssen: Elsevier.

29. Doornink N, Vogel J, Wipkink A, et al. (2006) Leidraad voor voedingskundigen bij kanker. Het hoofd-halscarcinoom (Manual for Nutritionists for Cancer. Head and Neck Cancer), 4th ed., pp. 54-71. Harlem: De Toorts.

30. Dawson ER, Morley SE, Robertson AG, et al. (2001) Increasing dietary supervision can reduce weight loss in oral cancer patients. Nutr Cancer 41, 70-74. 